\title{
Cytomegalovirus Infection in One Fetus with Hypoplasia of the Pons in a Diamniotic-Dichorionic Twin Pregnancy
}

\section{Introduction}

When a cytomegalovirus infection occurs within the first 16 weeks of pregnancy, leading to intrauterine transmission, it has severe implications for the fetus. The risk of a transplacental infection in the first trimester is $40 \%$, and $10-15 \%$ of these infected children show symptoms after birth. $80 \%$ of infected children suffer from severe impairment. We present a rare case of the infection of one fetus in a diamniotic-dichorionic twin pregnancy.

\section{Case report}

A 28-year-old Gravida 3, Para 1, Abortion 1 presented in week $21+1$ with a diamniotic-dichorionic twin pregnancy suspicious of a brain malformation in one fetus. She was sent to our unit with the diagnosis of mild ventriculomegaly and fetal growth retardation. Ultrasound examination showed that the weight estimation of the male fetus was below the $3^{\text {rd }}$ percentile. Detailed ultrasound examination of the CNS showed bilateral mild ventriculomegaly and narrowing of the corpus callosum ( $\mathbf{F i g}$. 1 ). The $3^{\text {rd }}$ ventricle measuring $4 \mathrm{~mm}\left(1.7 \mathrm{~mm}=97^{\text {th }}\right.$ percentile) was out of the normal range, according to the shallow irregular shape of the operculum. In addition to the wellknown changes of the CNS, we could also demonstrate changes of the infratentorial structures: The vermis showed a craniocaudal (cc) diameter of $13.9 \mathrm{~mm}(11.9 \pm 1.1)$, ap $8.7 \mathrm{~mm}$ (10.6 \pm 1.4$)$, circumference $40.7 \mathrm{~mm}(43.8 \pm 3.3)$, surface area 0.73 $\mathrm{mm}^{2}(0.9 \pm 0.2)$, demonstrating the reduction of the parenchyma of the vermis. The damage done by the virus also led to impairment of the regular lobulation of the vermis ( $>$ Fig. 2 ). We could also demonstrate a reduction in the size of the pons, not mentioned in the publication of Malinger about the sonographic findings in CMV infected fetuses (G. Malinger et al. AJNR 24: 28-32, 1/2003). We documented an ap diameter of $5.5 \mathrm{~mm}$, far below the $5^{\text {th }}$ percentile $(6.8 \mathrm{~mm})$. All findings were proved by MRI. The liver was of normal size, and calcifications in the liver or the brain could not be visualized at the first presentation.

Fetus II, a girl, did not show any symptoms.

The described ultrasound changes led to a screening for infections in the mother, showing a positive serum CMV-IgG titer and a CMV-IgM with high avidity, suspicious of a cytomegalovirus infection in early pregnancy. CMV infection of the compromised fetus was proved by amniocentesis (detec-

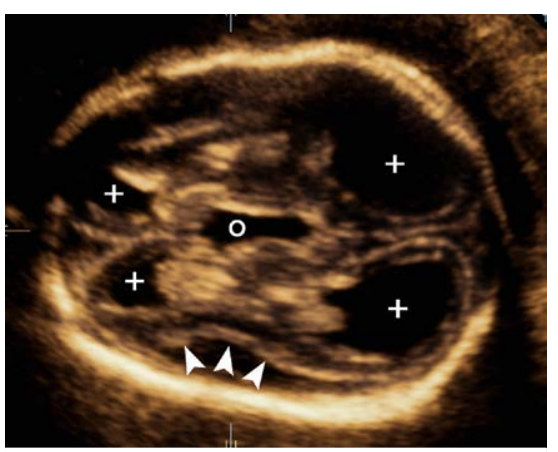

- Fig. 1 (21+2 weeks) shows dilated lateral ventricles $(+)$, dilated $3^{\text {rd }}$ ventricle $\left({ }^{\circ}\right)$ and a shallow shape of the operculum (arrowhead).

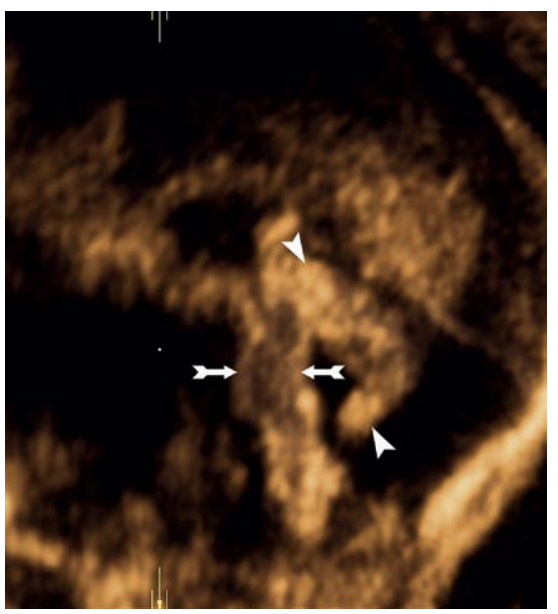

- Fig. 2 (21+2 weeks) shows narrowing of the diameter of the pons (arrowhead) and of the craniocaudal diameter of the vermis (arrow). Lobulation of the vermis cannot be displayed. tion of CMV-DNA). A genetic disorder was excluded by karyotyping.

Discussing the advantages and complications of the therapy with the mother, a decision was made to administer $8 \mathrm{~g}$ valaciclovir per day $(4 \times 2 \mathrm{~g})$ in off-label use, accompanied by blood sampling weekly to exclude myelosuppression.

In week $23+5$ the patient presented with preterm rupture of membranes of the leading, healthy girl. To avoid contractions, we administered atosiban, and antibiotic therapy was given to avoid infection of the membranes. In week 24 + 0 betamethasone was given and repeated after $24 \mathrm{~h}$ for lung maturation of the fetuses.

Growth retardation increased during the following weeks. The head circumference was stable below the $3^{\text {rd }}$ percentile, never reaching the criteria of microcephaly defined as -2(-3) SD. Unfortunately, the intracerebral lesions showed aggravation in size and intensity during the next weeks of gestation. The typical calcifications occurred in the brain and liver, and disruption of neuronal migration became visible ( $\triangleright$ Fig. 3). Hepatomegaly could be visualized, and the amniotic fluid increased to a polyhydramnios.

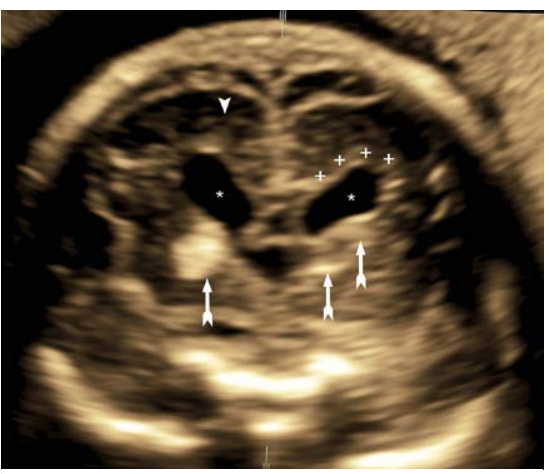

- Fig. $3(29+6$ weeks $)$ shows progression of all symptoms: dilated lateral ventricles $\left({ }^{*}\right)$, periventricular hyperechogenicity $(+)$, calcifications (arrow) and migration disorder (arrowhead) 
In week $30+1$ labor occurred leading to a secondary cesarean section. Upon request of the parents, comfort care was arranged for the sick newborn.

The infected boy (weight $1068 \mathrm{~g}$, length $35 \mathrm{~cm}$, head circumference $250 \mathrm{~mm}$, APGAR 5/5/5, ph 7.23) died on the first day after birth.

The girl was healthy suffering only from prematurity (weight $1220 \mathrm{~g}$ ). The CMV-DNA test of the girl proved the absence of a CMV infection. Histological examination of the placenta of the girl showed the expected chorioamnionitis due to the rupture of the membranes.

\section{Discussion}

Congenital cytomegalovirus infection is worldwide the most frequent viral congenital infection (0.2-2.2\%) followed by mental retardation and nonhereditary deafness in early childhood.

Seroconversion of CMV-IgG and the increasing CMV-IgM can prove an infection of the mother. Confirmation of the fetal infection is achieved by virus DNA in the amniotic fluid at least 8 weeks after the infection of the mother.

The damage done to the fetus is diagnosed based on ultrasonography: CNS (ventriculomegaly, intracranial calcifications, microcephaly, hydrocephaly), ascites, fetal growth retardation, hyperechogenic bowel, hepato-/splenomegaly, oligohydramnios and hydrops fetalis.

Nevertheless, the sensitivity to detect changes in CMV infection in the second trimester by ultrasound is very poor (15\%) (B. Guerra, Am J Obstet Gynecol 2008; 198: 380.e1-380.e7).

In our case of a rare congenital infection of a dichorionic-diamniotic twin pregnancy, it is interesting that the placenta of one fetus could prevent the transmission of the virus, while the placenta of the second fetus allowed the virus to pass the barrier. There are a few case reports about monochorionic twins describing the transmission of the virus through the placenta thereby infecting both fetuses (T. Lazzarotto et al., J Clin Virol 2008; 41(3): 192-197). In contrast, Yinon Y. concludes in a study of 20 twin pregnancies with primary or recurrent CMV infection that the placenta type cannot predict the pattern of infection in twins (Yinon Y. et al., Maternal Medicine, 2005, 14710528.2006.00854.x).

It has been proven that the placenta generates interferon, reducing the viral replication. Another protective mechanism is the placental production of HLA-G, which plays a key role in the protection of cells from CMV infection. Another theory describes the placenta as a nonspecific barrier against CMV. These results assume that the placenta might be the major factor for the transmission of the virus to the fetus.

The administration of CMV-specific antibodies to avoid transmission of the virus requires new evaluation. Valaciclovir at a high dosage $(8 \mathrm{~g} / \mathrm{d})$ might be more effective in the therapy of acute CMV infection with a low risk of side effects. In a pilot study the oral administration of high-dosage valaciclovir showed a significant reduction of the viral load (M. Leruez-Ville, Am J Obstet Gynecol 2016). The dosage given to the mother leads to therapeutic levels of $>17 \mathrm{mcmol} / \mathrm{L}$ in the fetal blood, resulting in a better fetal outcome after birth. In parallel, there is a need for a routine blood sampling, to avoid myelosuppression of the mother.

Finally, we have to confess that the therapeutic options are still uncertain. The decision about what to do needs detailed information and informed consent from the parents, especially with regard to the off-label use of the medication. Particularly in severely compromised fetuses, termination of the pregnancy should be discussed with the parents. The options should include individualized strategies like comfort care as in our case and demand an intensive dialog among all medical staff.

\section{Conflict of Interest}

The authors declare no conflict of interest.

\section{Authors}

\section{Reinhard Altmann ${ }^{1,2}$, Iris Scharnreitner ${ }^{1,2}$, Claudia Springer ${ }^{1,2}$, Franziska Pschebezin ${ }^{1,2}$, Wolfgang Arzt ${ }^{1,2}$ \\ Affiliations \\ 1 Department of Gynecology, Obstetrics and Gynecological Endocrinology, Kepler University Hospital, Johannes Kepler University Linz, Linz, Austria \\ 2 Department of Prenatale Medicine, Kepler University Hospital, Linz, Austria}

\section{Correspondence}

Dr. Reinhard Altmann

Johannes Kepler Universitat Linz

Prenatale Medicine

Krankenhausstrasse 26-30

4020 Linz

Austria

Tel.: + 43/576808423719

reinhard.altmann@kepleruniversitätsklinikum.at

\section{Bibliography}

DOI https://doi.org/10.1055/a-0591-5981

Ultrasound Int Open 2018; 4: E52-E53

(c) Georg Thieme Verlag KG Stuttgart · New York ISSN 2199-7152

\section{(요(1) $(2)$}

